\title{
Contribuições do Programa Mais Médicos ao desempenho de equipes de Saúde da Família na atenção à hipertensão e ao diabetes no Brasil, 2012 a 2015
}

\author{
Luiz Augusto Facchini, ${ }^{1}$ Alexandre de Souza Ramos Florencio, ${ }^{2}$ Bruno Pereira Nunes, ${ }^{1}$ \\ Marcilio Regis Melo Silva, ${ }^{3}$ Carlos Rosales, ${ }^{4}$ Gerardo Alfaro, ${ }^{5}$ Thiago Augusto Hernandes \\ Rocha $^{6}$ e Joaquín Molina ${ }^{4}$
}

Como citar

Facchini LA, Florencio ASR, Nunes BP, Silva MRM, Rosales C, Alfaro G, et al. Contribuições do Programa Mais Médicos ao desempenho de equipes de Saúde da Família na atenção à hipertensão e ao diabetes no Brasil, 2012 a 2015. Rev Panam Salud Publica. 2020;44:e63. https://doi.org/10.26633/RPSP.2020.63

RESUMO

Objetivo. Comparar as tendências temporais de acesso e utilização de serviços para controle de hipertensão arterial sistêmica (HAS) e diabetes mellitus (DM) em equipes com e sem a participação de profissionais do Programa Mais Médicos (PMM).

Métodos. Utilizou-se um delineamento analítico-descritivo, com comparação entre equipes participantes do PMM (intervenção) e não participantes, em uma abordagem quase-experimental em série temporal. Comparouse o desempenho de uma amostra de 30000 equipes da Estratégia Saúde da Família em 2012 e 20000 equipes em 2015. Os padrões nos dois grupos foram analisados pela técnica de diferença-em-diferença com estratificação por região geopolítica, porte populacional e perfil municipal. A média semestral de atendimentos foi estimada pela divisão do número total de consultas de DM e HAS realizadas por médicos e enfermeiros em determinada equipe de saúde pelo total de usuários com DM/HAS cadastrados no mesmo local e período. Uma média de consultas/usuário maior do que 14,2 para DM e 10,8 para HAS foi considerada como outlier, sendo as equipes com essas médias excluídas das análises.

Resultados. A análise de diferença-em-diferença indicou melhor desempenho na oferta de consulta para DM $(P<0,001)$ e HAS $(P<0,001)$ entre as equipes com PMM em comparação àquelas sem o programa. $O$ efeito foi mais pronunciado nas regiões Norte e Nordeste, em municípios com mais de $20 \%$ da população em extrema pobreza e em municípios de todos os portes populacionais.

Conclusões. Os resultados mostram que o PMM amplia o acesso e a utilização dos serviços de saúde.

Palavras-chave Avaliação de programas e projetos de saúde; atenção primária à saúde; hipertensão; diabetes mellitus; Brasil.

\footnotetext{
Universidade Federal de Pelotas, Departamento de Medicina Social, Pelotas (RS), Brasil

2 Organización Panamericana de la Salud/Organización Mundial de la Salud, (OPS/OMS), Manágua, Nicarágua.

3 Ministério da Saúde, Brasília (DF), Brasil.

4 Organização Pan-Americana da Saúde/Organização Mundial da Saúde (OPAS/OMS), Brasília (DF), Brasil.
}

\footnotetext{
Organización Panamericana de la Salud/Organización Mundial de la Salud (OPS/OMS), Cidade do Panamá, Panamá.

6 Organização Pan-Americana da Saúde/Organização Mundial da Saúde (OPAS/OMS), Unidade Técnica de Doenças Transmissíveis e Análise de Situação de Saúde. $\square$ hernandthi@paho.org
} 
Lançada em 1994, a Estratégia Saúde da Família (ESF) impulsionou a universalização e a qualificação da atenção primária à saúde (APS) no Brasil, mas sempre enfrentou dificuldades em sua expansão por conta da escassez de médicos, particularmente em áreas remotas e vulneráveis (1). Em 2013, em decorrência da insuficiência histórica de profissionais formados e da má distribuição de profissionais no território nacional, o Brasil dispunha de 1,8 médico por 1000 habitantes - muito abaixo da referência de 2,7 médicos por 1000 habitantes, média dos países integrantes da Organização para a Cooperação e Desenvolvimento Econômico (OECD). Além disso, em 22 estados brasileiros a situação era ainda pior, indicando uma elevada concentração de médicos e a presença de iniquidades internas no país quanto à distribuição de médicos (2).

Uma das soluções para enfrentar esse desafio foi o Programa Mais Médicos (PMM), lançado em 2013 pelo Ministério da Saúde. O PMM é um dos mais ousados projetos de extensão equitativa de cobertura da APS no mundo $(2,3)$. Com abrangência nacional e presença em contextos muito diversos, o PMM articulou a provisão emergencial de um grande contingente de médicos, chegando a 17 mil profissionais. Enfocou também a educação permanente e a formação médica para o SUS (4-6).

A implantação do PMM ocorreu em um contexto de desenvolvimento e consolidação de outras iniciativas de governança que incentivaram a APS nos últimos 10 anos no Brasil. O PMM teve interação com o Programa de Melhoria do Acesso e da Qualidade da Atenção Básica (PMAQ) (7), com o Programa de Infraestrutura e Requalificação de Unidades Básicas de Saúde (REQUALIFICA UBS) e com os programas de formação e educação permanente de profissionais de saúde do SUS, desenvolvidos por intermédio da Universidade Aberta do SUS (UNASUS) (8). Enquanto iniciativa de governança (9), o PMM estabeleceu regras, normas e decisões, criou e distribuiu papéis e responsabilidades a atores sociais e promoveu interações e interconexões em combinação com diversas políticas dirigidas à APS, com particular interesse na redução de disparidades e iniquidades nacionais e regionais (2).

A importância do PMM reside em sua abrangência nacional, que promove justiça social ao estender o direito à saúde em favor de populações mais vulneráveis e incentiva o desenvolvimento e a maturidade institucional do SUS (4, 10-14). O PMM também contribui para a organização de funções e estruturas na gestão regional e local da APS, particularmente quanto a definição e regulação de trabalhadores e profissionais de saúde, aquisição e distribuição de medicamentos e tecnologias, geração e disseminação de informações e construção, reforma e ampliação de UBS, viabilizando os meios para financiar a prestação de serviços de saúde à população $(6,14,15)$.

A avaliação das características e dos efeitos do PMM vem ocorrendo desde o início do programa, cobrindo extensa gama de temas, reflexões e metodologias (16). Em um primeiro momento, aspectos relativos à distribuição dos profissionais do PMM no território nacional ganharam destaque, com resultados indicando um forte alcance equitativo na cobertura de populações que não dispunham de assistência adequada à saúde, ou que dispunham dessa assistência de modo muito precário e irregular $(12,17,18)$. Existem diversos estudos sobre o impacto do PMM no desempenho dos serviços, incluindo a melhoria do acesso e da qualidade da atenção e também da situação de saúde da população atendida $(4,6,14,16)$.
Apesar dos referidos avanços, a avaliação da efetividade do PMM em melhorar equitativamente o desempenho de equipes da ESF continua sendo um desafio complexo - separar qual parte de um determinado resultado se deve ao PMM é uma tarefa desafiadora, em função do caráter multideterminado dos resultados em saúde. Diante desse desafio, o presente estudo propôs a utilização do Marco de Monitoramento e Avaliação desenvolvido pela Organização Pan-Americana da Saúde/Organização Mundial da Saúde (OPAS/OMS) para examinar uma série de indicadores relacionados ao PMM (19). No contexto do Marco, o objetivo deste trabalho foi comparar as tendências temporais de acesso e utilização de serviços para controle de hipertensão arterial sistêmica (HAS) e diabetes mellitus (DM) em equipes com e sem a participação de profissionais do PMM.

\section{MATERIAIS E MÉTODOS}

O presente estudo utilizou um delineamento analíticodescritivo para exame do desempenho dos indicadores de atenção a HAS e DM. Foram utilizados grupos de intervenção e comparação definidos em função da participação no PMM, com abordagem quase-experimental (20) em série temporal (21).

\section{Contexto: unidades de análise, indicadores e bases de dados}

O efeito do PMM no desempenho das equipes da ESF foi avaliado pela apreciação dos resultados provenientes da série temporal 2012-2015 do Sistema de Informação da Atenção Básica (SIAB), vigente à época. A unidade de análise considerada foram as equipes de ESF, separadas entre aquelas com profissionais do PMM e aquelas sem profissionais do PMM. Para o exame de uma eventual modificação de efeito decorrente do PMM, as equipes que receberam profissionais do PMM a partir de 2013 foram identificadas como "com PMM" mesmo nos anos anteriores à implantação do programa.

O número de equipes avaliadas foi definido pela aplicação dos critérios de consistência e completude. A análise da contribuição do PMM ao desempenho da ESF foi realizada em uma amostra de, aproximadamente, 30000 equipes a partir de 2012 20885 equipes sem PMM mais 10310 equipes que receberam médicos do PMM a partir de 2012. Isso sugere que 30\% do total de equipes em 2012 tinham problemas e descontinuidade na oferta regular de consultas médicas à população (tabela 1). Tendo por objetivo a priorização da qualidade das informações reportadas em 2015, foram analisadas em torno de 20000 equipes que faziam a transmissão regular de dados.

Seguindo o Marco de Monitoramento e Avaliação $(4,20)$, foram comparados o padrão e a tendência temporal de acesso e a utilização de serviços mediante indicadores de média de consultas de controle de DM e de HAS em equipes com e sem a participação de profissionais do PMM, para os períodos antes (2012-2013) e depois (2014-2015) da implantação do programa. Para fins de definição da média semestral de atendimentos por DM e HAS, adotou-se o seguinte entendimento: a média é estimada pela divisão do número total de consultas de DM e HAS realizadas por médicos e enfermeiros em determinada equipe de saúde a cada semestre pelo total de residentes com DM e HAS cadastrados no mesmo local e período. O indicador reflete o acesso bem-sucedido a consultas por pessoas com DM e HAS considerando a população cadastrada. Uma média de consultas 
TABELA 1. Número de equipes da Estratégia Saúde da Família estratificadas por vinculação ao Projeto Mais Médicos, Brasil, 2012 a 2015

\begin{tabular}{|c|c|c|c|c|c|c|c|c|}
\hline \multirow{2}{*}{$\begin{array}{l}\text { Indicador: } \\
\text { consultas médicas }\end{array}$} & \multicolumn{2}{|c|}{2012} & \multicolumn{2}{|c|}{2013} & \multicolumn{2}{|c|}{2014} & \multicolumn{2}{|c|}{2015} \\
\hline & $1^{\circ}$ semestre & $2^{\circ}$ semestre & $1^{\circ}$ semestre & $2^{\circ}$ semestre & $1^{\circ}$ semestre & $2^{\circ}$ semestre & $1^{\circ}$ semestre & $2^{\circ}$ semestre \\
\hline \multicolumn{9}{|l|}{ Diabetes } \\
\hline Sem PMM & 20744 & 20722 & 21738 & 21559 & 18657 & 18490 & 13569 & 13531 \\
\hline \multicolumn{9}{|l|}{ Hipertensão } \\
\hline Sem PMM & 20788 & 20753 & 21707 & 21558 & 18591 & 18461 & 13569 & 13522 \\
\hline
\end{tabular}

por habitante maior do que 14,2 para DM e 10,8 para HAS foi considerada como outlier, sendo as equipes com essas médias excluídas das análises. Esses valores foram baseados nos parâmetros estabelecidos na avaliação do PMAQ para atendimento de pessoas com DM (de 3,0 a 7,1) e HAS (de 2,0 a 5,4). Paras não restringir demasiadamente o número de equipes, e considerando que algumas equipes podem ter produção acima do esperado, multiplicamos o valor máximo por 2, a fim de obter um limite conservador na definição de outliers. Para DM, as exclusões por outliers representaram 783 equipes no segundo semestre de 2012 e 578 equipes no segundo semestre de 2015. Para HAS, as exclusões por outliers representaram 812 equipes no segundo semestre de 2012 e 620 no segundo semestre de 2015. Valores de outliers mais extremos foram observados para equipes sem o PMM no período.

O SIAB permite cadastrar as equipes da ESF e repassar incentivos financeiros aos municípios, além de acompanhar as ações e os resultados das atividades realizadas na ESF, incluindo produção e composição das equipes. O sistema, que em 2016 foi convertido em e-SUS Atenção Básica (e-SUS AB), reunia cerca de 118 milhões de usuários potenciais nas equipes cadastradas. Apesar de sua relevância, sempre apresentou problemas de implantação, completude, envio e consolidação da base de dados. Dependendo dos critérios de completude e consistência aplicáveis à informação, a amostra analisada poderia apresentar uma expressiva variabilidade em relação ao universo de equipes possível, considerando-se o intervalo de tempo analisado. Assim, utilizaram-se duas estratégias combinadas para a seleção das equipes expostas e não expostas ao PMM junto ao SIAB: envio regular de dados ao SIAB na série 2012-2015 por pelo menos 10 meses ao ano e preenchimento dos critérios de consistência do PMAQ especificamente para os indicadores de controle de DM e HASS em equipes com e sem a participação de profissionais do PMM. Os indicadores do PMAQ foram usados como proxy de atividade da equipe (equipe ativa e enviando os dados regularmente), uma vez que, sem envio das informações, o repasse de recursos variáveis vinculados à avaliação do PMAQ poderia ser suspenso.

\section{Análise de dados}

Em consonância com as diretrizes metodológicas da OMS (22), e valorizando as dimensões no Marco de Monitoramento e Avaliação do PMM/OPAS (4, 20), foi utilizada para avaliação uma matriz conceitual hierárquica (figura 1). A matriz guarda profunda sintonia com as categorias de análise estrutura, processo e resultados, definidas por Donabedian (23); representa graficamente os propósitos do PMM e sistematiza categorias e dimensões utilizadas para descrever, predizer e discutir a intervenção em estudo $(4,21,24)$.

A matriz conceitual situa o PMM como uma iniciativa de governança do SUS (25), direcionada à melhoria do acesso, da cobertura, da efetividade e da qualidade da ESF em diferentes contextos sociais. Operacionalmente, o modelo define três níveis hierárquicos, sendo o primeiro, ou distal, o que reúne os determinantes de maior relevância contextual, também denominados macrodeterminantes. Nesse nível estão situadas características e divisões territoriais e grande agregados sociais - por exemplo, país, região geopolítica e município (porte populacional e perfil municipal) - que sintetizam padrões históricos de condição de vida da população, de acesso à riqueza, ao desenvolvimento social e à infraestrutura de serviços públicos, em especial da rede de saúde e de atenção primária à saúde de regiões e municípios do país. Nesse nível também se encontram as características sociais, ocupacionais, culturais, demográficas, comportamentais e de saúde da população, conformando grupos prioritários e vulneráveis para a resposta em saúde dos serviços. Para entender a relação entre população e serviços de saúde é conveniente considerar que a população não é um todo homogêneo, tampouco o mero somatório de seus indivíduos. As desigualdades sociais se expressam em diferentes populações e territórios.

No segundo nível, ou intermediário, o modelo localiza as políticas sociais, como por exemplo, o PMM, que expressa iniciativa, ou ação de governança, implementada para modificar o efeito de macrodeterminantes estruturais e históricos sobre o desempenho da APS. O PMM, enquanto decisão política e de governança, guarda relação com diversos insumos estratégicos do sistema de saúde, como, por exemplo, o direcionamento de financiamento para ampliar de modo significativo a disponibilidade de força de trabalho, particularmente de médicos, em localidades pequenas, mais pobres e remotas, mas também nas periferias de grandes centros urbanos. A iniciativa também interage com outras políticas direcionadas à melhoria da informação, da educação permanente dos profissionais, do acesso a medicamentos e tecnologias. Os insumos estratégicos objetivam qualificar a estrutura e o processo de trabalho dos serviços básicos (23), situados em nível hierárquico proximal, que influenciam diretamente o desempenho da $\operatorname{APS}(25,26)$. O desempenho dos serviços de APS, considerado o desfecho do presente modelo teórico, contempla indicadores de acesso e utilização de serviços, também podendo incluir qualidade do cuidado, satisfação do usuário, situação de saúde e proteção ao risco financeiro (27). 
FIGURA 1. Modelo teórico para analisar a relação do Programa Mais Médicos com o desempenho do sistema de saúde

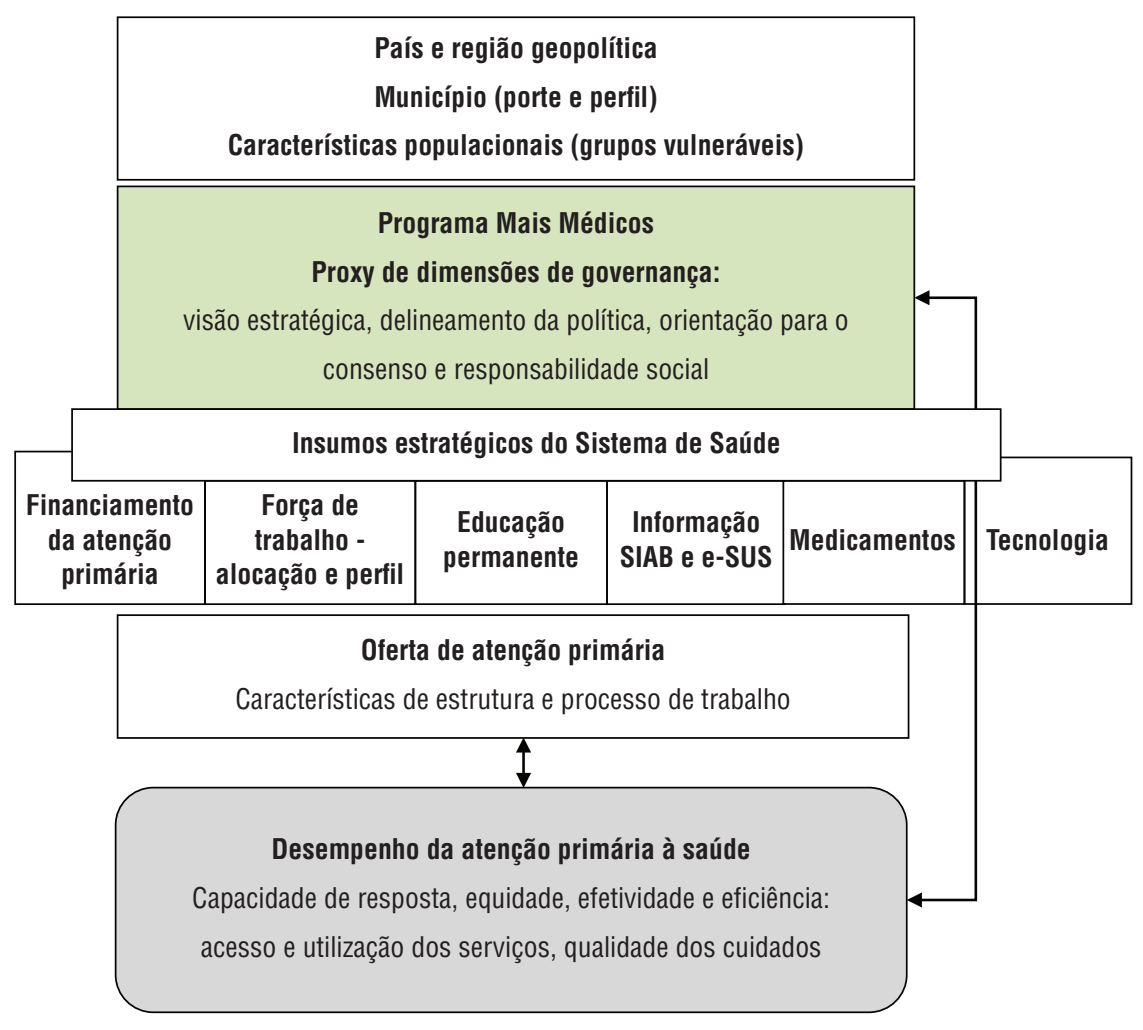

Além de aspectos próprios dos serviços, o desempenho também expressa os resultados da tomada de decisão política e de governança do sistema de saúde, em dimensões como capacidade de resposta, equidade, efetividade e eficiência (25). O modelo em questão foi utilizado para balizamento do processo de análise de dados, segundo a lógica de relacionamento entre os diferentes elementos que perpassam o processo de oferta de cuidado em APS. Nesse sentido, buscou-se evidenciar de que forma o PMM pode impactar a dimensão de resultado que aparece na figura 1.

Segundo o modelo teórico-avaliativo proposto na figura 1 , o exame dos dados envolveu o cálculo de média e desvio-padrão para obtenção das medidas síntese por semestre do período entre 2012 e 2015 para os indicadores de consultas para DM e HAS. Posteriormente, foram realizadas análises estatísticas de diferença (análise de diferença-em-diferença) para entendimento da evolução dos indicadores segundo equipes com e sem profissionais do PMM.

Para estimar mudanças atribuíveis ao PMM, utilizou-se a análise de diferença-em-diferença, que permite estabelecer uma relação de inferência causal de intervenções não randomizadas (21, 28-31) estratificadas por região geopolítica, porte populacional e perfil do município. A análise baseia-se na comparação das diferenças entre o grupo que recebeu determinada intervenção (equipes com PMM) e o grupo não exposto à intervenção, ou contrafactual (equipes sem PMM), antes e depois do início da avaliação. Teoricamente, essa diferença das diferenças consegue melhorar a inferência causal pois permite controlar variáveis não mensuradas e comuns aos grupos, sendo sua utilização cada vez mais frequente, principalmente para a avaliação de políticas de saúde (31-33). Valores $P$ foram obtidos por teste t através da regressão linear. As análises foram realizadas com o número de observação após exclusão dos outliers. Os "n" apresentados nos resultados refletem esse processo.

O estudo utilizou informações de bases de dados anonimizadas, que caracterizam perfis de atendimentos e de serviços de saúde, portanto sem a necessidade de aprovação por comitê de ética.

\section{RESULTADOS}

Com o PMM, a oferta de consultas de DM e HAS na APS cresceu no período estudado, passando de 33,1\% em 2012 para 36,2\% em 2016. Multiplicando-se o número de equipes de ESF estudadas neste artigo por 4000 habitantes, encontra-se um conjunto de mais de 36 milhões de pessoas com acesso regular a consultas médicas em equipes atendidas por profissionais do programa, em um universo de mais de 100 milhões de pessoas atendidas.

O número de equipes sem PMM, para ambos os indicadores, variou de aproximadamente 20000 em 2012 para 13000 em 2015. As equipes com PMM estavam mais presentes nos municípios com extrema pobreza e nas regiões Norte e Nordeste. Para as outras características estratificadas, não foram observadas diferenças percentuais significativas.

No período de 2012 a 2015, a rede básica de saúde manteve um padrão estável de 3 consultas médicas por DM e 2 consultas por HAS por indivíduo a cada semestre, com ligeiras diferenças entre equipes com e sem profissionais do PMM. Entretanto, a implantação do PMM melhorou o desempenho de equipes 
FIGURA 2. Tendência dos indicadores selecionados para A) diabetes e B) hipertensão em equipes sem e com Programa Mais Médicos, Brasil, 2012 a 2015

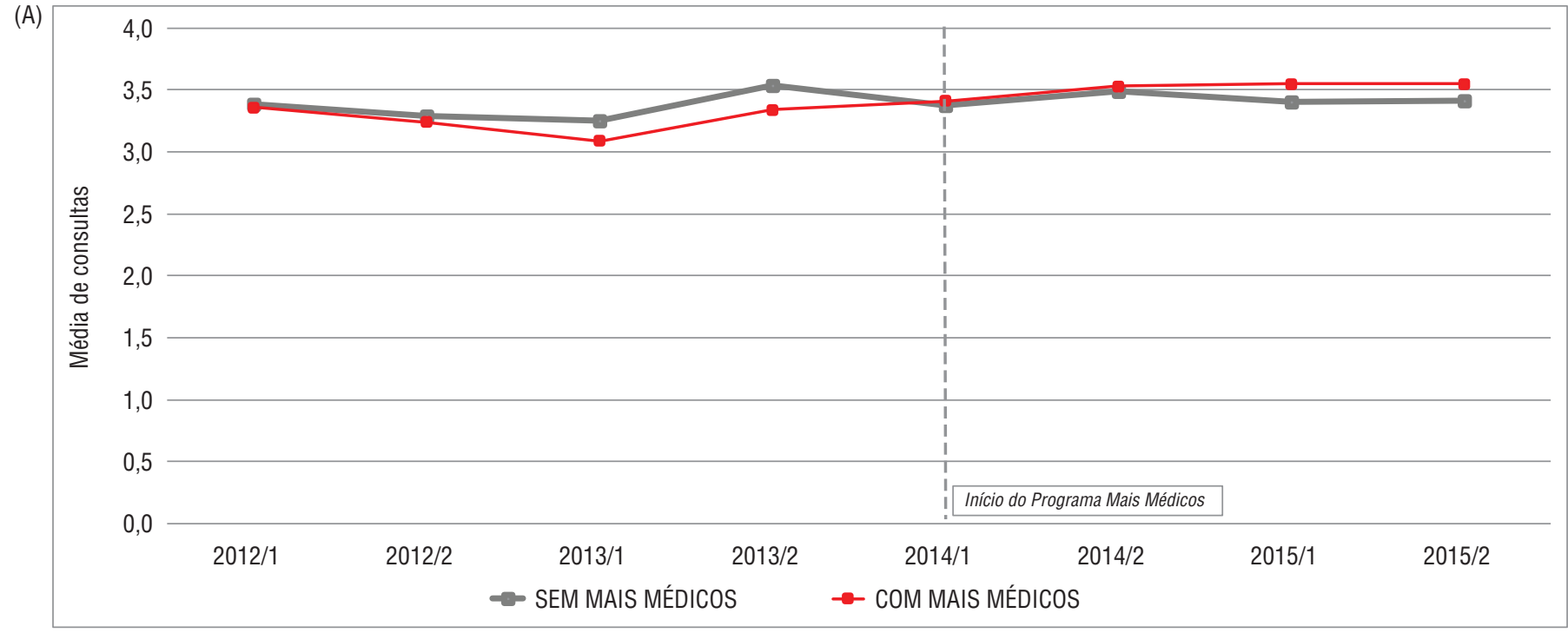

(B)

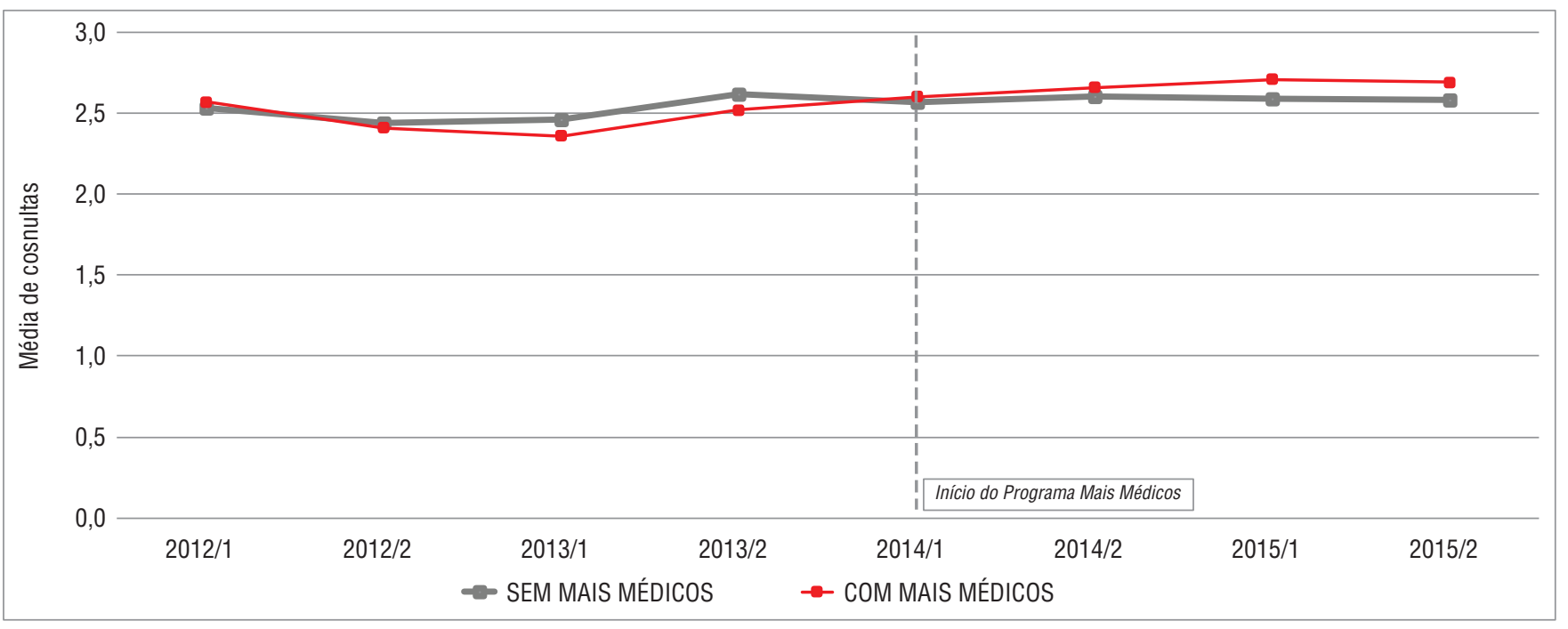

a As equipes que ingressaram no Programa Mais Médicos a partir de 2014, ano de implantação do programa, são consideradas como "com mais médicos" mesmo antes desse ano.

que receberam profissionais do programa. Na série temporal 2012-2015, a análise de diferença-em-diferença demonstrou uma ligeira melhora no desempenho quanto à tendência de consulta médica para DM $(P<0,001)$ e HAS $(P<0,001)$ entre as equipes com PMM em comparação àquelas sem o programa (contrafactual). Assim, em média, equipes com PMM realizaram, aproximadamente, 0,15 consultas/semestre a mais por habitante (figura 2 e tabela 2). As diferenças observadas foram de 0,188 consultas para DM e 0,140 consultas para HAS, ambas em favor das equipes com PMM.

Tanto para HAS como DM, o PMM apresentou efeito estatisticamente significativo no aumento médio de consultas para a comparação entre regiões (destaque para as regiões Norte e Nordeste), perfis municipais (municípios com mais de $20 \%$ da população vivendo em extrema pobreza e municípios) e portes populacionais (tabela 3).

\section{DISCUSSÃO}

Confirmando evidências de diversos estudos (2, 6, 11, 17), nossos achados indicam um efeito sistemático do PMM na melhoria dos indicadores estudados. Isso ocorreu apesar da localização dos profissionais em áreas mais pobres, mais remotas e com populações mais vulneráveis. Embora pequenas, as diferenças favoráveis ao PMM foram regulares, com os profissionais do programa garantindo a ampliação do acesso e da cobertura das ações de saúde investigadas. Destaca-se que os indicadores utilizados refletem a média semestral de consultas por habitante, sendo esperada a ocorrência de pequenas variações (quando existem), mas não de mudanças expressivas, dada a complexidade da medida e de seu significado.

A presença de médicos com dedicação exclusiva em 36,2\% das equipes estudadas aumentou a proporção de consultas de 
TABELA 2. Variação anual e análise de diferença-em-diferença para os indicadores, Brasil, 2012 a 2015

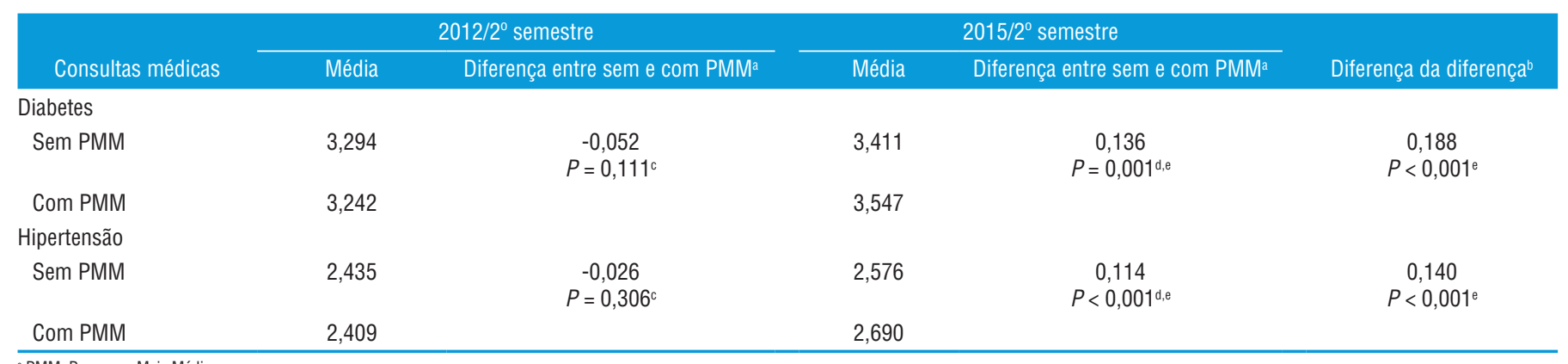

a PMM: Programa Mais Médicos.

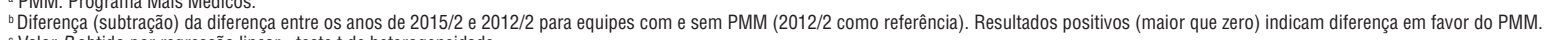

'Valor $P$ obtido por regressão linear - teste t de heterogeneidade.

e Associação estatisticamente significativa $(P<0.05)$.

TABELA 3. Análise de diferença-em-diferença para consultas médicas conforme região geopolítica, perfil municipal e porte populacional, Brasil, 2012 a 2015

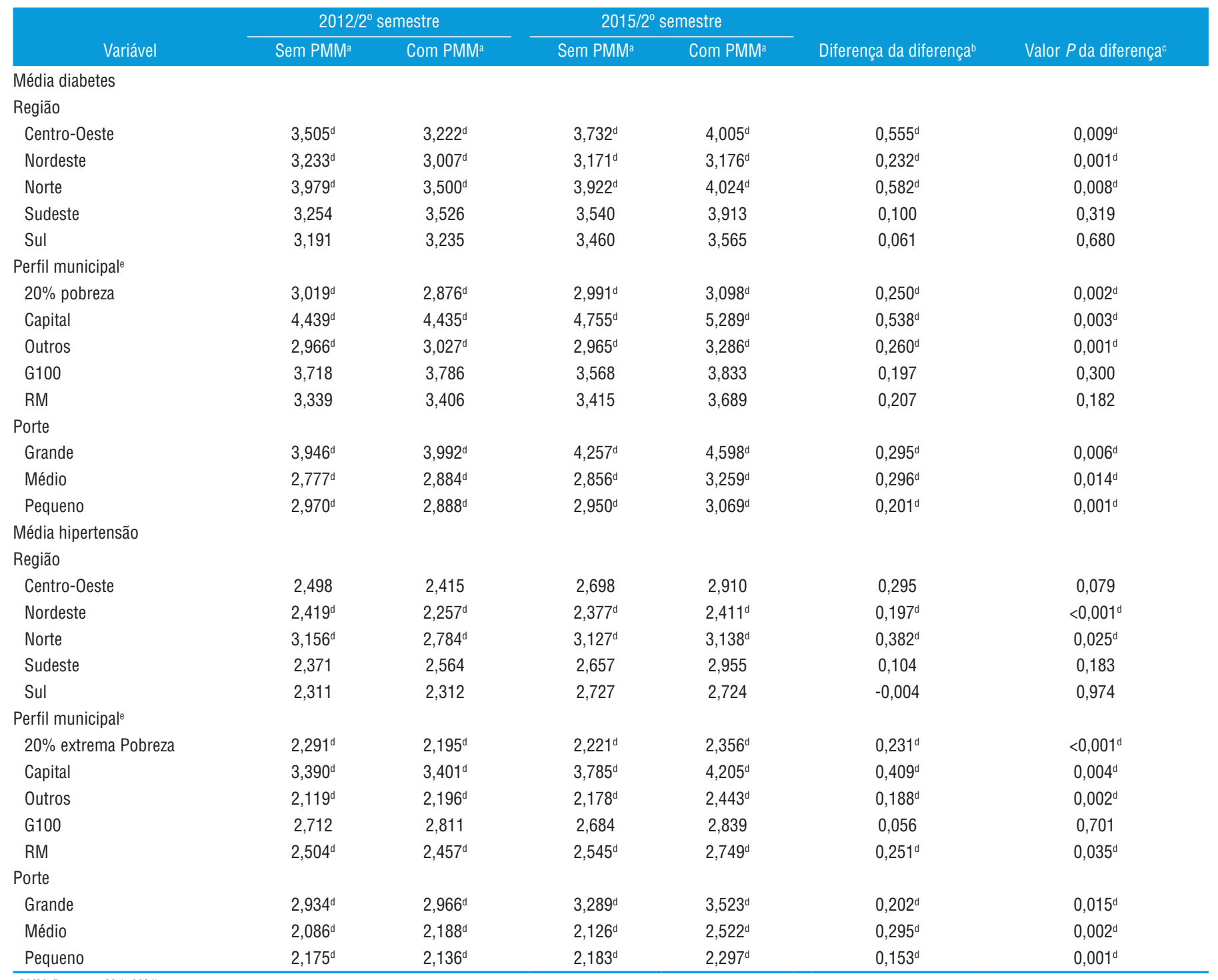

a PMM: Programa Mais Médicos.

${ }^{b}$ Diferença (subtração) da diferença entre os anos de 2015/2 e 2012/2 para equipes com e sem PMM (2012/2 como referência). Resultados positivos (maior que zero) indicam diferença em favor do PMM. c Valor $P$ obtido por regressão linear - teste t de heterogeneidade. Valor $P$ da diferença entre equipes com e sem PMM em cada período.

¿Associação estatisticamente significativa $(P<0,05)$.

e Perfis prioritários para implantação do PMM: $20 \%$ pobreza = municípios com $20 \%$ ou mais da população vivendo em extrema pobreza; capitais; outros = demais municípios; $6100=100$ municípios mais pobres do país; RM = municípios pertencentes a regiôes metropolitanas. 
cuidado continuado, garantindo o acesso e a longitudinalidade do cuidado a 36 milhões de pessoas antes inadequadamente assistidas. Esse achado é central no entendimento do efeito sistêmico do PMM no Sistema Único de Saúde (SUS) no Brasil, com redução de desigualdades na atenção à saúde.

As evidências indicam que serviços APS que orientam suas ações com base nos determinantes sociais da saúde (34), quando vinculados ao PMM, facilitam o acesso a ações essenciais de promoção da saúde, prevenção de complicações inerentes a DM e HAS e cuidado desses agravos. As recomendações que emer-gem das evidências direcionam-se ao fortalecimento da ESF (5), com vantagens no sentido de organizar o SUS e as ações e cuidados de saúde nos serviços de APS com foco nas pessoas e no território de abrangência. Nesse contexto, o PMM significou uma contribuição valiosa para a garantia de acesso universal e equitativo a ações de saúde na APS.

O PMM não é imune às contradições e aos obstáculos relativos ao SUS e sua rede de serviços. Assim, uma das reclamações de usuários atendidos no PMM refere-se ao sistema de refe-rência para atenção especializada e realização de exames (6). Os serviços de APS continuam carentes de melhor estrutura, incremento de tecnologias e capacidade de gestão do cuidado mediante compartilhamento de tarefas entre os membros das equipes multiprofissionais, aspectos que exigem investimentos continuados em infraestrutura e educação permanente de pro-fissionais e gestores.

Outro desafio do PMM decorre da heterogeneidade da população, mesmo em áreas mais pobres e relativamente homogêneas. As carências sociais podem ser de grande mag-nitude, dificultando o cuidado dispensado pelos profissionais de saúde. Nesse sentido, é importante fortalecer as abordagens intersetoriais dirigidas aos determinantes sociais da saúde, com classificação de risco de usuários da rede básica e gestão popu-lacional da saúde no território de abrangência do serviço.

Independentemente da relevância dos achados e de eventuais fragilidades do PMM, os resultados estão inseridos em um contexto de limitações inerentes à avaliação de programas de saúde (20). As dificuldades em processar a informação do SIAB foram marcantes. Os problemas de fidedignidade dos dados registrados prejudicam a interpretação da natureza do atendimento. Nesse sentido, a continuidade da utilização dos sistemas de informação da APS e sua divulgação para equi-pes de saúde, gestores e público acadêmico é essencial para melhorar o registro de dados e avaliar o efeito das mudanças para o e-SUS em indicadores sob monitoramento e avaliação.

Outra limitação no exame do impacto do PMM no desempenho das equipes de APS resulta do curto período transcorrido desde o início da intervenção, ou seja, da provisão e educação permanente de profissionais. Entretanto, a grande abrangência e complexidade do PMM, com suas múltiplas intervenções arti-culadas, aumentam a possibilidade de sucesso de sua avaliação de impacto.

Em função da escassez de informações sobre indicadores de processo, procedimentos e cuidados, mais adequados para ava-liar a qualidade da APS, recomenda-se a continuidade de estudos de impacto para melhor compreensão do alcance do PMM no país. Por fim, a análise de dados utilizada poderia ter incluído mais variáveis para melhor entendimento dos achados. Apesar de sistêmico, o efeito do programa deve ser avaliado em bases de dados com maior poder para permitir análises com controle de confusão e especificidade da ação dos profissionais do programa.

O PMM é a maior iniciativa do Estado brasileiro direcionada à provisão de profissionais médicos para A PS $(5,10)$. O pro-grama mostrou capacidade de melhorar o padrão de consultas médicas nas equipes em que seus profissionais atuaram, alcan-çando, e mesmo superando, o padrão das melhores equipes de APS do país que não contavam com profissionais do PMM. O estudo confirma as evidências de que o PMM amplia a efe-tividade do SUS e da APS, garantindo o acesso prioritário a expressivas parcelas da população $(6,10,12)$. Os achados do presente estudo servem de estímulo para consolidar a provisão emergencial de médicos como uma importante estratégia dedicada a abordar a iniqui-dade na distribuição de médicos, que aflige diversos países do mundo e, principalmente, das Américas.

Contribuições dos autores. LAF, ASRF, BPM, MRMS, CR, GA, TAHR participaram do processo de revisão dos estudos de caso, escrita, revisão e aprovação final do manuscrito. JM parti-cipou do processo de revisão dos estudos de caso e da escrita e revisão do manuscrito. Todos os autores aprovaram a versão final.

Conflito de interesses. Nada declarado pelos autores.

Declaração. As opiniões expressas no manuscrito são de responsabilidade exclusiva dos autores e não refletem neces-sariamente a opinião ou política da RPSP/PAJPH ou da Organização Pan-Americana da Saúde (OPAS).

\section{REFERÊNCIAS}

1. Rocha TAH, da Silva NC, Thomaz EBAF, Queiroz RCS, de Souza MR, Lein A, et al. Primary health care and cervical cancer mortality rates in Brazil: a longitudinal ecological study. J Ambul Care Manage. 2017;40(Suppl 2):S24-34.

2. Ministério da Saúde. Programa Mais Médicos - dois anos: mais saúde para os brasileiros. Brasília: Secretaria de Gestão do Trabalho e da Educação na Saúde; 2015. Disponível em: http:/ /bvsms .saude.gov.br/bvs/publicacoes/programa_mais_medicos_dois _anos.pdf Acessado em agosto de 2019.

3. Brasil. Lei 12 871/2013. Brasília: Diário Oficial da União; 2013. Disponível em: http://www.planalto.gov.br/ccivil_03/_ato2011 -2014/2013/Lei/L12871.htm Acessado em agosto de 2019.

4. Molina J, Tasca R, Suárez J. Monitoring and evaluation of the PAHO/WHO cooperation project, the Mais Médicos (More
Doctors) Program: a mid-term assessment. Cienc Saude Colet. 2016;21(9):2925-33.

5. Facchini LA, Batista SR, da Silva Jr AG, Giovanella L. The Mais Médicos (More Doctors) Program: assessments and prospects. Cienc Saude Colet. 2016;21(9):2652.

6. Santos LMP, Oliveira A, Trindade JS, Barreto ICHC, Palmeira PA, Comes $\mathrm{Y}$, et al. Implementation research: towards universal health coverage with more doctors in Brazil. Bull World Health Organ. 2017;95(2):103-12.

7. Ministério da Saúde. Programa Nacional de Melhoria do Acesso e da Qualidade da Atenção Básica (PMAQ): manual instrutivo. Brasília: Secretaria de Atenção à Saúde; 2012. Disponível em: http://189.28.128.100/dab/docs/publicacoes/geral/manual _instrutivo_pmaq_site.pdf Acessado em agosto de 2019. 
8. Thumé E, Wachs LS, Soares MU, Cubas MR, Fassa MEG, Tomasi E, et al. Physicians' reflections on the personal learning process and the significance of distance learning in family health. Cienc Saude Colet. 2016;21(9):2807-14.

9. Brinkerhoff DW, Bossert TJ. Health governance: concepts, experience, and programming options. Washington: USAID; 2008. Disponível em: https://www.hfgproject.org/wp-content /uploads/2015/02/Health-Governance-Concepts-Experience-and -Programming-Options.pdf Acessado em agosto de 2019.

10. Molina J, Tasca R, Suárez J, Kemper ES. More Doctors Programme and the strengthening of Primary Health Care in Brazil: reflections from the monitoring and evaluation of the More Doctors Cooperation Project. Qual Prim Care. 2017;25(2):50-4.

11. Giovanella L, de Mendonça MHM, Fausto MCR, de Almeida PF, Bousquat A, Lima JG. Emergency supply of doctors by the Mais Médicos (More Doctors) Program and the quality of the structure of primary health care facilities. Cienc Saude Colet. 2016;21(9):2697-708.

12. Campos GWS, Pereira Júnior N. Primary care in Brazil, and the Mais Médicos (More Doctors) Program in the Unified Health System: achievements and limits. Cienc Saude Colet. 2016;21(9):2655-63.

13. Barbosa ACQ, Amaral PV, Francesconi GV, Rosales C, Kemper ES, da Silva NC, et al. More Doctors recruitment program: a new approach to overcome inequalities in human resources. Rev Panam Salud Publica. 2018;42(1):e185.

14. Carrillo B, Feres J. Provider supply, utilization, and infant health: evidence from a physician distribution policy. Am Econ J Econ Policy. 2019;11(3):156-96.

15. World Health Organization (WHO). The World Health Report 2000: health systems: improving performance. Genebra: WHO; 2000. Disponível em: https://www.who.int/whr/2000/en/whr00_en.pdf Acessado em agosto de 2019.

16. Kemper ES, Mendonça AVM, de Sousa MF. The Mais Médicos (More Doctors) Program: panorama of the scientific output. Cienc Saude Colet. 2016;21(9):2785-96.

17. Girardi SN, Carvalho CL, Pierantoni CR, Costa JO, van Stralen ACS, Lauar TV, et al. Assessment of the scope of practice of physicians participating in the Mais Médicos (More Doctors) Program, and associated factors. Cienc Saude Colet. 2016;21(9):2739-48.

18. Santos LMP, Costa AM, Girardi SN. Mais Medicos Program: an effective action to reduce health inequities in Brazil. Cienc Saude Colet. 2015;20(11):3547-52.

19. Organização Pan-Americana da Saúde (OPAS)/Organização Mundial da Saúde (OMS). Marco para o monitoramento e avaliação do Projeto de Cooperação Mais Médicos da OPAS/OMS. Brasília: OPAS; 2015.

20. White H. A contribution to current debates in impact evaluation. Evaluation. 2010;16(2):159-64.

21. Steele F. Multilevel models for longitudinal data. J R Stat Soc Ser A Stat Soc. 2008;171(1):5-19.

22. World Health Organization (WHO). Handbook on health inequality monitoring: with a special focus on low- and middle-income countries. Genebra: WHO; 2013. Disponível em: http://apps.who .int/iris/bitstream/handle/10665/85345/9789241548632_eng.pdf? sequence=1 Acessado em agosto de 2019 .

23. Donabedian A. The quality of care: how can it be assessed? JAMA. 1988;260(12):1743-8

24. Bickman L. The functions of program theory. New Dir Eval. 1987;1987(33):5-18.

25. Mikkelsen-Lopez I, Wyss K, de Savigny D. An approach to addressing governance from a health system framework perspective. BMC Int Health Hum Rights. 2011;11(1):13.

26. Facchini LA, Piccini RX, Tomasi E, Thumé E, Silveira DS, Siqueira FV, et al. Desempenho do PSF no Sul e no Nordeste do Brasil: avaliação institucional e epidemiológica da Atenção Básica à Saúde. Cienc Saude Colet. 2006;11(3):669-81.

27. Roberts MJ, Hsiao W, Berman P, Reich MR. Getting health reform right: a guide to improving performance and equity. Oxford: Oxford University Press; 2003.

28. Antunes JLF, Cardoso MRA. Uso da análise de séries temporais em estudos epidemiológicos. Epidemiol Serv Saude. 2015;24(3): $565-76$.

29. Basu S, Meghani A, Siddiqi A. Evaluating the health impact of large-scale public policy changes: classical and novel approaches. Annu Rev Public Health. 2017;38(1):351-70.

30. Craig P, Katikireddi SV, Leyland A, Popham F. Natural experiments: an overview of methods, approaches, and contributions to public health intervention research. Annu Rev Public Health 2017;38(1):39-56.

31. Reeves BC, Wells GA, Waddington H. Quasi-experimental study designs series-paper 5: a checklist for classifying studies evaluating the effects on health interventions - a taxonomy without labels. J Clin Epidemiol. 2017;89(1):30-42.

32. Hu Y, van Lenthe FJ, Judge K, Lahelma E, Costa G, de Gelder R, et al. Did the English strategy reduce inequalities in health? A difference-in-difference analysis comparing England with three other European countries. BMC Public Health. 2016;16(1):865.

33. Rosenthal MB, Alidina S, Friedberg MW, Singer SJ, Eastman D, Li $\mathrm{Z}$, et al. A difference-in-difference analysis of changes in quality, utilization and cost following the Colorado multi-payer patient-centered medical home pilot. J Gen Intern Med. 2016;31(3):289-96.

34. Wei X, Li H, Yang N, Wong SY, Chong MC, Shi L, et al. Changes in the perceived quality of primary care in Shanghai and Shenzhen, China: a difference-in-difference analysis. Bull World Health Organ. 2015;93(6):407-16.

Manuscrito recebido em 20 de novembro de 2018. Aceito em versão revisada em 6 de agosto de 2019 . 


\section{Contributions of the More Doctors Program to the performance of Family Health teams concerning hypertension and diabetes care in Brazil, 2012 to 2015}

ABSTRACT Objective. To compare temporal trends in access and utilization of systemic arterial hypertension (SAH) and diabetes mellitus (DM) services provided by teams with or without physicians from the More Doctors Program (PMM).

Method. An analytical-descriptive design was used, with comparison of teams that joined the PMM (intervention) vs. non-participants, using a quasi-experimental time series approach. The study compared the performance of a sample of 30000 Family Health Strategy teams in 2012 and 20000 teams in 2015. The patterns in both groups were analyzed using the difference-in-difference technique with stratification according to geopolitical region, population size, and municipal profile. The mean number of consultations per semester was estimated by dividing the total number of DM and HAS consultations provided by physicians and nurses in a given health care team by the total number of users with DM/HAS registered in the same location and period. A mean number of consultations/user > 14.2 for DM and > 10.8 for HAS was considered as an outlier, and thus the teams with these means were excluded from the analyses.

Results. The difference-in-difference analysis indicated better performance in the provision of DM $(P<0.001)$ and SAH $(P<0.001)$ services among PMM teams vs. non-PMM teams. The effect was more pronounced in the North and Northeast regions, in municipalities with more than $20 \%$ of the population living in extreme poverty, and in municipalities of all population sizes.

Conclusions. The results show that the PMM increased access and utilization of health care services.

Keywords

Program evaluation; primary health care: hypertension; diabetes mellitus; Brazil.

\section{Contribuciones del Programa Mais Médicos al desempeño de los equipos de salud de la familia en el cuidado de la hipertensión y la diabetes en Brasil, 2012-2015}

RESUMEN

Objetivo. Comparar las tendencias temporales del acceso y de la utilización de los servicios para el control de la hipertensión arterial sistémica (HAS) y la diabetes mellitus (DM) realizado por los equipos de salud de la familia que trabajan solos y por los que cuentan con la participación de profesionales del Programa Mais Médicos (PMM).

Métodos. Se utilizó un diseño analítico y descriptivo, con comparación entre los equipos con participantes del PMM (intervención) y sin ellos, con un método cuasiexperimental de series temporales. Se comparó el desempeño de una muestra de 30000 equipos que trabajaron dentro del marco de la estrategia de salud de la familia en el 2012 y de 20000 equipos en el 2015. Se analizaron los patrones observados en ambos grupos con la técnica de doble diferencia con estratificación por zona geopolítica, tamaño de la población y perfil municipal. Se estimó la media semestral de casos atendidos con la división del número total de consultas por DM y HAS a cargo de médicos y miembros del personal de enfermería de un determinado equipo de salud por el total de usuarios con DM y HAS registrados en el mismo lugar y período. Se consideró que una media de consultas por DM por usuario mayor de 14,2 y por HAS mayor de 10,8 era un valor atípico y, por lo tanto, los equipos con esas medias se excluyeron de los análisis.

Resultados. El análisis de doble diferencia indicó un mejor desempeño en la atención de consultas por DM $(P<0,001)$ y por HAS $(P<0,001)$ a cargo de los equipos con participantes del PMM en comparación con los que trabajaron sin ese programa. El efecto fue más pronunciado en las regiones norte y nordeste, en los municipios con más de $20 \%$ de la población en condiciones de extrema pobreza y en municipios de todos los tamaños de población.

Conclusiones. Los resultados muestran que el PMM amplía el acceso y la utilización de los servicios de salud.

Palabras clave Evaluación de programas y proyectos de salud; atención primaria de salud; hipertensión; diabetes mellitus; Brasil. 